\title{
NUMERICAL APPROACH FOR BENDING STRESS ASCERTAINMENT IN BEAM THEORY CONSIDERING EFFECTS OF ELASTIC SHEAR LAG
}

\author{
Matthias KRAUS \\ Faculty of Civil Engineering, Bauhaus-University Weimar, Marienstr. 13, 99423, Weimar, Germany \\ Received 14 August 2016; accepted 26 November 2016

\begin{abstract}
In beam theory, normal stresses due to bending moments are usually determined with regard to Bernoullis hypothesis of the cross section staying in a plane state for reasons of simplicity. As a result of this fundamental assumption, the influence of shear on the distribution of normal stresses is not taken into account. However, for cross sections showing wide flanges, as they are commonly applied in bridge engineering for instance, the effect can usually not be disregarded. For the sake of capturing, stiffness oriented numerical approaches allowing the determination of the shear deformation behaviour of cross sections are applied and the shear influence is introduced into the normal stress calculation. This is being exemplarily shown for normal stresses due to bending moments $M_{y}$.
\end{abstract}

Keywords: beam theory, cross sections, shear deformations, shear lag, bending stresses, finite elements.

\section{Introduction}

The internal moment $\mathrm{M}_{\mathrm{y}}$ of a beam, being the bending moment about the principal axis y of the cross section, is linked to normal stresses $\sigma_{\mathrm{x}}$. The calculation of these stresses is usually performed using the relationship:

$$
\sigma_{x}=\frac{M_{y}}{I_{y}} \cdot z,
$$

where $I_{y}$ is the principal moment of inertia and $z$ the principal axis as shown in Figure 1. The normal stresses are also connected to shear stresses $\tau$ for reasons of equilibrium, which are summarized to the internal force $V_{z}$. Since Eq. (1) requires the cross section to stay in a plane state, the influence of the corresponding shear stresses and strains, respectively, is not displayed in emerging stress states for $\sigma_{x}$. Figure 1 shows an example of a single-symmetric cross section. The stress distribution calculated with Eq. (1) leads to a constant course in the upper flange as shown in Figure 1(b).

However, in a more detailed perspective, shear strains will influence the normal stress distribution specified as elastic shear lag, see Figure 1(c). Due to the elasticity and deformation behaviour, respectively, caused by shear straining, the outer edges of the flange do not contribute to the load transfer as strong as the connection region to the web does. Therefore stresses concentrate in the middle of the flange and decrease to the outer edges. The flange stress calculated with Eq. (1) and displayed in Figure 1(b) represents a mean value of the stress state closer to reality as shown in Figure 1(c).

The described effect can mainly be observed for cross sections with wide flanges, that is large a flange width in comparison to member length. It becomes clear that the stress distribution in the flange directly depends on the cross section shape as well as the structural system. In addition, acting loads have an decisive influence as well.

For capturing the changing stress distribution in the transversal flange direction, cross section parts (plates) have to be modelled using plate and shell theories, respectively. This goes along with quite a large 
M. Kraus. Numerical approach for bending stress ascertainment in beam theory considering effects ...

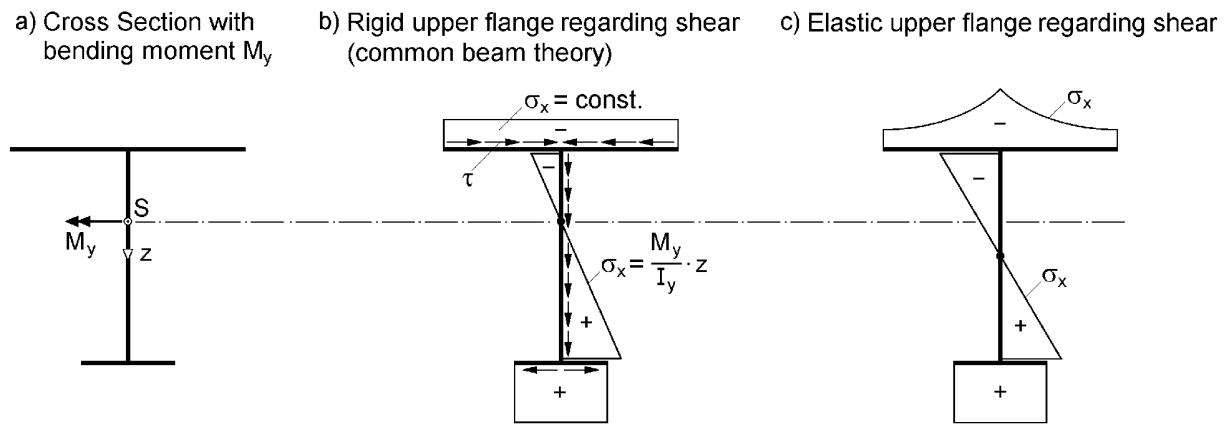

Fig. 1. Distribution of Stresses due to $M_{y}$ according to (Kindmann, Krahwinkel 2016)

effort and regarding classical beam theory, an application is not even straightforwardly possible. For that reason, the so-called effective width for elastic shear lag has been derived in the past and incorporated into Eurocode 3 (EN 1993-1-5: 2006 + AC: 2009). The principles are shown in Figure 2, corresponding derivations and analyses can be found in (Schmidt, Peil 1976), (Schmidt et al. 1979) or (Sedlacek, Bild 1990) for example. The changing distribution of $\sigma_{x}$ in the flange is replaced by a constant course exhibiting $\sigma_{\mathrm{x}}=$ max. $\sigma_{\mathrm{x}}$ and at the same time, the real flange width is reduced to the effective width $b_{\text {eff }}$ thus both stress distributions lead to the same resultant force in the flange. Taking the effective width into account for the modelling of the cross section and the determination of the principle moment of inertia $I_{y}$, stresses can now be calculated according to the classical beam theory using Eq. (1) and leading to the maximum stress in the flange.
In the following, an approach for accouting normal stress distributions considering the elastic shear lag and avoiding the effective width identification is being presented. The basis is a cross section model using the finite element method (FEM), which allows the determination of shear deformations and which is particularly suitable for computer-aided implementations. Especially for thin-walled cross sections, the deformations can be calculated with small effort using one-dimensional finite elements (line model, see Fig. 3). For that reason, they are focused on here.

\section{Stiffness relationship for shear deformations}

In the publications (Kindmann, Kraus 2011) and (Kraus 2005) stiffness relationships are derived in detail on the basis of FEM, allowing a calculation of shear deformations $u_{V}$ of a cross section. Figure 4 shows the corresponding element with two nodes and the nodal

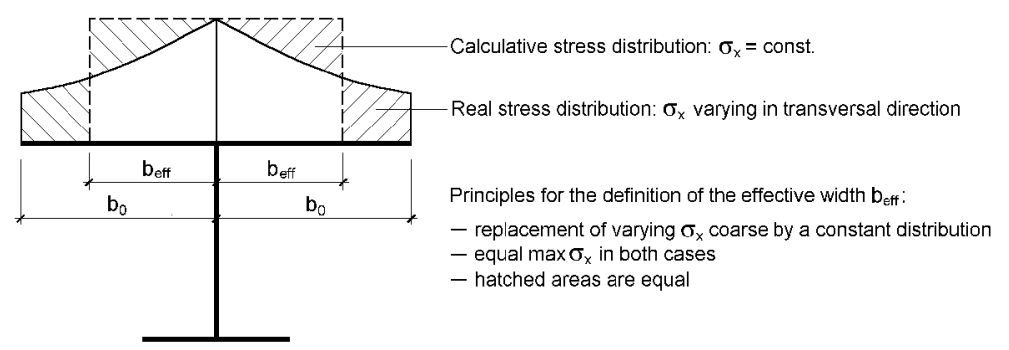

Fig. 2. Model of effective width according to (Kindmann, Krahwinkel 2016)

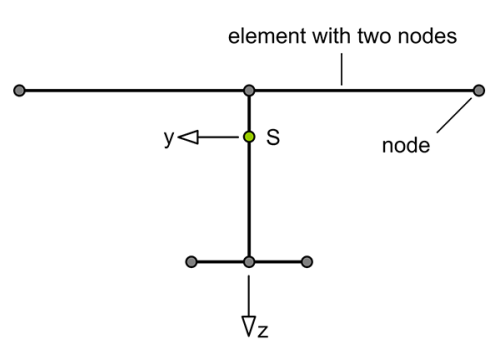

Discretisation of the cross section

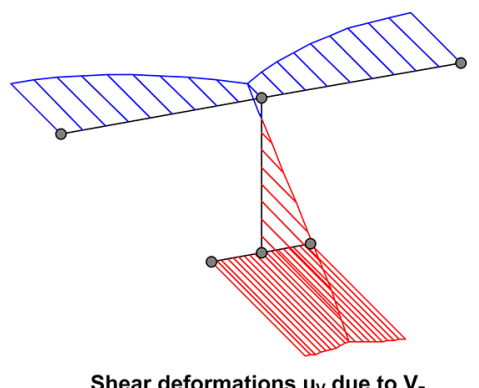

Shear deformations $u_{v}$ due to $V_{z}$

Fig. 3. FE-Model of a cross section and shear deformations due to $V_{z}$ 


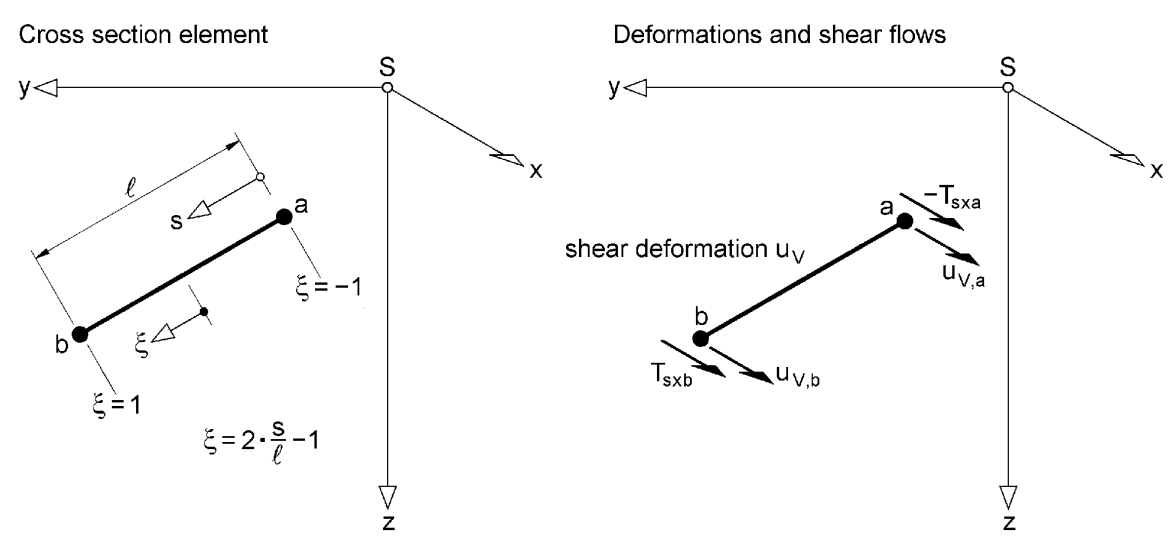

Fig. 4. Cross section element with two nodes, degrees of freedom and boundary shear flows

degrees of freedom $u_{V}$ as well as the shear flows $T_{s x}=$ $\tau_{s x} \cdot t$ at the element edges, respectively, where $t$ is the thickness of the element (plate). For shear forces $V_{z}$ (going along with the bending moment $M_{y}$ ) and related shear deformations, the following element stiffness relationship is specified:

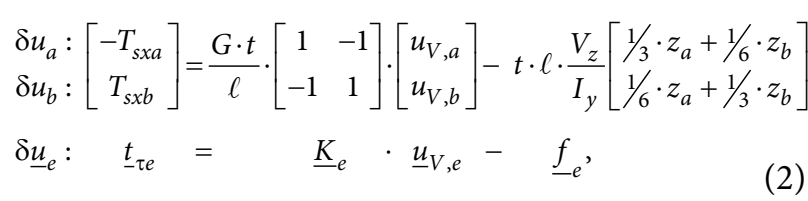
where: $G$ - Shear modulus; $\underline{t}_{\tau e}$ - Element vector of shear flows; $\underline{K}_{e}$ - Element stiffness matrix; $\underline{u}_{V, e}$ - Element vector of shear deformations; $f_{e}$ - Element load vector.

Within beam theory, shear deformations $u_{V}$ due to $V_{z}$ show a cubic course for thin walled, rectangular plates at maximum. For that reason, cubic Lagrangian polynomials as shape functions as shown in Figure 5 constitute the basis of the element derivation, leading to an element with four nodes at first. Through a static liquefaction and the elimination of internal nodes, the element can be transformed into the one with two nodes as described in Eq. (2).

For the calculation of shear deformations of a cross section, which prevalently consists of several rectangular plates, modelling using the finite elements of Eq. (2) is undertaken as shown in Figure 3. At every position, where cross section parts are connected to each other or intersect, as well as at all end points of the plates, a node is arranged. The assembling of the global stiffness relationship (by element stiffness) is provided by formulating shear flow equilibrium at the nodes incorporating shear flows of the element edges.

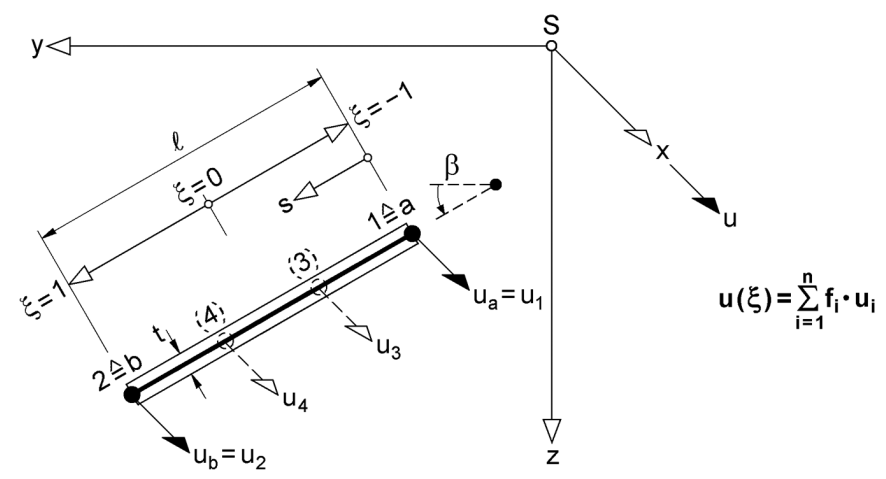

a) linearly varying approach

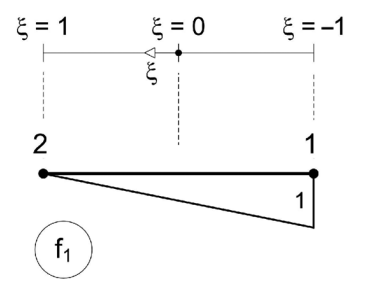

b) cubic approach

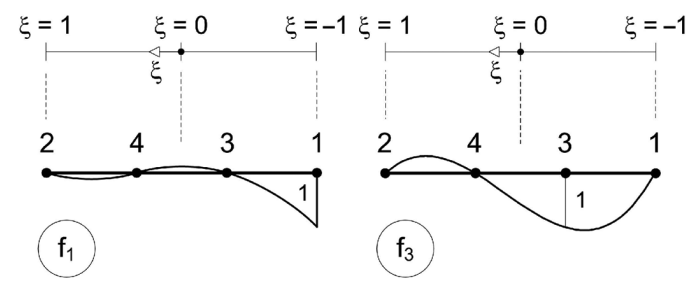

Fig. 5. Lagrangian functions for line elements 
Formally, the global stiffness relationship of cross sections is set up in the known manner as for instance conducted for beam structures, leading to the following equation system:

$$
\underline{K} \cdot \underline{u}_{V}=\underline{f} .
$$

This equation system cannot be solved for the time being since it is singular due to a missing support of the cross section. For this reason, an arbitrary node i has to be held nondisplaceably in the u-direction:

$$
u_{V, i}=0 \text {. }
$$

This boundary condition is included in the equation system (3) evolved. Although the solution vector of the shear deformations does not contain the exact node displacements then, the values being determined only differ from the actual deformations by a rigidbody ratio. For reasons of indication, these shear deformations are specified with a superscripted cross as $\underline{\underline{u}}$.

The rigid-body ratio can be determined regarding the condition of positive and negative shear-warping parts $u_{V}$ of the cross section to be equal. If they were not equal, shear deformations and strains, respectively, would be connected to a mean deformation u (regarding the entire cross section) corresponding to the displacement of an internal normal force. This would not agree to pure bending considered here. Thus, the rigidbody ratio $u_{V, k}$ is gained by the following equation as a weighted sum of shear deformations regarding the cross section area A:

$$
u_{V, k}=\frac{\int_{A} \bar{u}_{V} \cdot d A}{A} .
$$

In the sense of FEM, the integration of Eq. (5) is replaced by a summation over the number of elements $n_{e}$ and then solved per element. In doing so, the functional course of $u_{V}$ can be described using the cubic Lagrangian polynomials. For the integration of Eq. (5), the assumption of constant element thickness $t$ and the transformation of the differential ds into the local coordinate system $(d s=L / 2 \cdot d \xi,-1 \leq \xi \leq 1)$ lead to:

$$
\begin{aligned}
& \int_{A} \bar{u}_{V} \cdot d A=\sum_{j=1}^{n_{e}}\left[t \cdot \frac{\ell}{2} \cdot \int_{-1}^{1} \underline{f} \cdot \underline{u}_{V} \cdot d \xi\right]_{j}= \\
& \sum_{j=1}^{n_{e}}\left[\frac{1}{8} \cdot t \cdot \ell \cdot\left(\bar{u}_{V, 1}+\bar{u}_{V, 2}+3 \cdot \bar{u}_{V, 3}+3 \cdot \bar{u}_{V, 4}\right)\right]_{j} .
\end{aligned}
$$

In the context of the aforementioned static liquefaction, shear deformations $u_{3}$ and $u_{4}$ are described by the boundary nodal displacements $u_{1}$ and $u_{2}$. The following relationship is given by (Kraus 2005):

$$
\begin{aligned}
& {\left[\begin{array}{l}
u_{V, 3} \\
u_{V, 4}
\end{array}\right]=\frac{\ell^{2}}{G} \cdot \frac{V_{z}}{I_{y}} \cdot\left[\begin{array}{cc}
5 / 81 & 4 / 81 \\
4 / 81 & 5 / 81
\end{array}\right] \cdot\left[\begin{array}{l}
z_{1} \\
z_{2}
\end{array}\right]+} \\
& {\left[\begin{array}{cc}
2 / 3 & 1 / 3 \\
1 / 3 & 2 / 3
\end{array}\right] \cdot\left[\begin{array}{l}
u_{V, 1} \\
u_{V, 2}
\end{array}\right] .}
\end{aligned}
$$

By introducing Eq. (7) into Eq. (6), the shear displacements of internal nodes can be eliminated as follows:

$$
\int_{A} \bar{u}_{V} \cdot d A=\sum_{j=1}^{n_{e}}\left[\begin{array}{l}
\frac{t \cdot \ell}{2} \cdot\left(\bar{u}_{V, a}+\bar{u}_{V, b}\right)+ \\
\frac{t \cdot \ell^{3} \cdot V_{z}}{24 \cdot G \cdot I_{y}} \cdot\left(z_{a}+z_{b}\right)
\end{array}\right]_{j}
$$

In compliance to Figure 1, the boundary nodes are now designated by indices $\mathrm{a}$ and $\mathrm{b}$ instead of 1 and 2. The rigid-body ratio $u_{V, k}$ can be determined using Eq. (5) and the actual displacement of the node i produces to:

$$
u_{V, i}=\bar{u}_{V, i}-u_{V, k} .
$$

\section{Determination of normal stresses}

Regarding small deformations, displacements $u$ of beam theory can be described using the following approximation:

$$
u(x, y, z)=z \cdot \varphi_{y}(x)+u_{V}(x, y, z) .
$$

The first term corresponds to classical beam theory neglecting shear deformations (Bernoulli hypotheses), where longitudinal displacements u are described by means of rotation about the $y$ axis in a linearized manner as shown in Figure 6(a). Since secondary shear deformations are neglected in this description, they are covered by the second term with $u_{V}$, see Figure 6(b).

Normal stresses are being described by theory of elasticity (Hookes law) and strains by the derivative of displacement. With the introduction of Eq. (10), the following relationship can be stated:

$$
\sigma_{x}=E \cdot \varepsilon_{x}=E \cdot \partial u / \partial x=E \cdot\left(z \cdot \varphi_{y}^{\prime}+u_{V}^{\prime}\right) .
$$

The bending moment as resultant of the normal stresses is defined by the following integration:

$$
M_{y}=\int_{A} \sigma_{x} \cdot z \cdot d A .
$$

By introducing Eq. (11) into (12), a formulation for the derivate of the cross section rotation can be stated:

$$
M_{y}=\int_{A} E \cdot\left(z \cdot \varphi_{y}^{\prime}+u_{V}^{\prime}\right) \cdot z \cdot d A=
$$




$$
\begin{aligned}
& E \cdot I_{y} \cdot \varphi_{y}^{\prime}+E \cdot \int_{A} u_{V}^{\prime} \cdot z \cdot d A \Rightarrow \\
& \varphi_{y}^{\prime}=\frac{M_{y}}{E \cdot I_{y}}-\frac{1}{I_{y}} \cdot \int_{A} u_{V}^{\prime} \cdot z \cdot d A .
\end{aligned}
$$

Thus, the corresponding deformation value can be replaced in Eq. (11) leading to an expression for $\sigma_{x}$, depending on the bending moment $M_{y}$ as well as the shear deformations $u_{V}$ :

$$
\sigma_{x}=\frac{M_{y}}{I_{y}} \cdot z-\frac{E}{I_{y}} \cdot \int_{A} u_{V}^{\prime} \cdot z \cdot d A \cdot z+E \cdot u_{V}^{\prime} .
$$

Regarding $u_{V}^{\prime}=\partial u_{V} / \partial x$, Eq. (14) contains the derivate of shear displacements with respect to $\mathrm{x}$. Therefore normal stresses do not depend on the actual shear displacements, but on their change in longitu- dinal beam direction. Due to the fact that the finite element formulations for cross sections of the previous chapter do not cover the longitudinal direction, this has to be captured in a different appropriate manner.

Shear displacements are linked to shear stresses and therefore to the corresponding shear force $V_{z}$ in beam theory. The variation of shear forces in longitudinal beam direction is associated to an according variation of shear displacements as shown in Figure 7. Thus the derivate of shear displacements $u_{V}^{\prime}$ in Eq. (14) corresponds to the diversification of shear force. In case of distributed loads $q_{z}$, shear forces reveal a linear distribution in longitudinal beam direction and shear displacements will also change linearly then. At the same time, the ratio of shear force to shear deformation does not change within the member length.

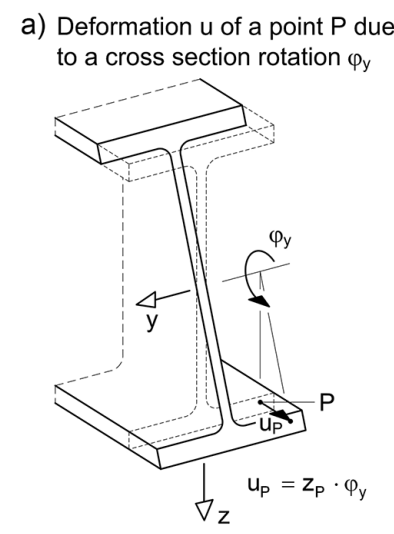

b) Deformations u due to shear action
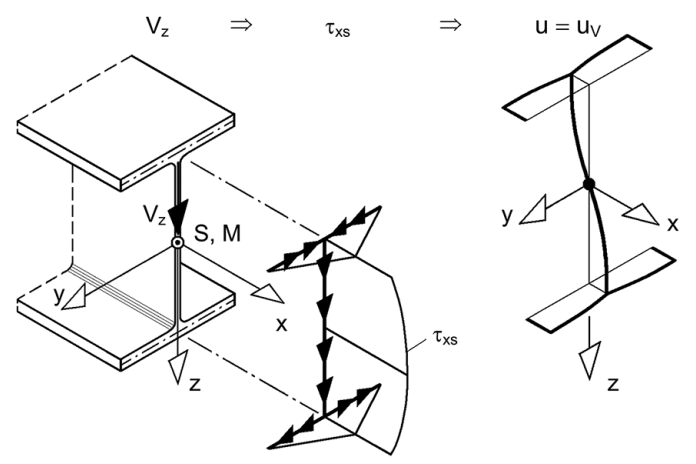

Fig. 6. Description of the deformation $u$

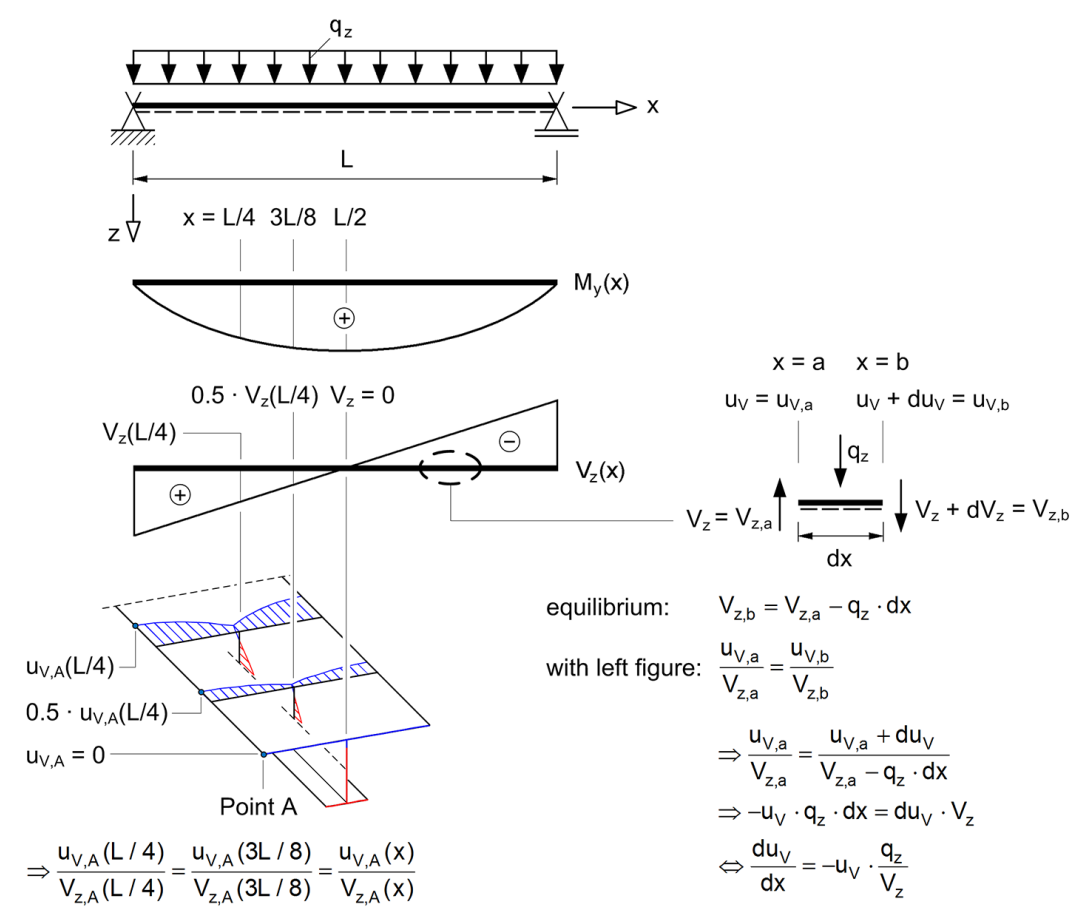

Fig. 7. Relationship between shear force and shear displacements in beam theory 
In Figure 7, a relationship to describe $u_{V}^{\prime}$ is derived with the help of a differential element $d x$, the assumption of a constant distributed load $q_{z}$ and equilibrium in $\mathrm{z}$ direction:

$$
u_{V}^{\prime}=\frac{\partial u_{V}}{\partial x}=-u_{V} \cdot \frac{q_{z}}{V_{z}} .
$$

Introducing into Eq. (14), normal stress can be described as:

$$
\sigma_{x}=\frac{M_{y}}{I_{y}} \cdot z+\frac{E}{I_{y}} \cdot \frac{q_{z}}{V_{z}} \cdot \int_{A} u_{V} \cdot z \cdot d A \cdot z-E \cdot \frac{q_{z}}{V_{z}} \cdot u_{V} .
$$

According to the previous chapter and regarding the solution of the FEM equation system, displacements are ascertained at the element nodes. For their use in Eq. (16), the integration is replaced by a summation over the number of elements and the functional courses of $u_{V}$ and $z$, respectively, are approximated using Lagrangian polynomials according to Figure 5:

$$
\begin{aligned}
& \sigma_{x}=\frac{M_{y}}{I_{y}} \cdot z+\frac{E}{I_{y}} \cdot \frac{q_{z}}{V_{z}} . \\
& \sum_{j=1}^{n_{e}}\left[t \cdot \frac{\ell}{2} \cdot \int_{-1}^{1} \underline{z}^{T} \cdot \underline{f}^{T} \cdot \underline{f} \cdot \underline{u}_{V} \cdot d \xi\right]_{j} \cdot z-E \cdot \frac{q_{z}}{V_{z}} \cdot u_{V} \cdot
\end{aligned}
$$

Since shear displacements indicate a cubic course for straight-lined elements, corresponding polynomials are introduced for $\mathrm{u}_{\mathrm{V}}$ in Eq. (17) and regarding $\mathrm{z}$ ordinates, linear polynomials are sufficient. Solving the integration in Eq. (17) leads to the following expression:

$$
\begin{aligned}
& t \cdot \frac{\ell}{2} \cdot \int_{-1}^{1} \underline{z}^{T} \cdot \underline{f}^{T} \cdot \underline{f} \cdot \underline{u}_{V} \cdot d \xi=\frac{t \cdot \ell}{120} \cdot\left[\begin{array}{ll}
z_{1} & z_{2}
\end{array}\right] . \\
& {\left[\begin{array}{cccc}
13 & 2 & 36 & 9 \\
2 & 13 & 9 & 36
\end{array}\right] \cdot\left[\begin{array}{l}
u_{V, 1} \\
u_{V, 2} \\
u_{V, 3} \\
u_{V, 4}
\end{array}\right] .}
\end{aligned}
$$

Taking into account Eq. (6), the internal nodal values, that is nodes 3 and 4 , can be eliminated. Through introduction into Eq. (18) and consideration of Eq. (17), a formulation for determining normal stresses $\sigma_{x}$ is maintained:

$\sigma_{x}=\frac{M_{y}}{I_{y}} \cdot z-E \cdot \frac{q_{z}}{V_{z}} \cdot u_{V}+$

$\frac{E}{I_{y}} \cdot \frac{q_{z}}{V_{z}} \cdot \sum_{j=1}^{n_{e}}\left[\begin{array}{l}\frac{t \cdot \ell}{6} \cdot\left[\begin{array}{l}2 \cdot z_{a}+z_{b} \\ z_{a}+2 \cdot z_{b}\end{array}\right] \cdot\left[\begin{array}{l}u_{V, a} \\ u_{V, b}\end{array}\right]+ \\ \frac{t \cdot \ell^{3} \cdot V_{z}}{180 \cdot G \cdot I_{y}} \cdot\left(4 \cdot z_{a}^{2}+7 \cdot z_{a} \cdot z_{b}+4 \cdot z_{b}^{2}\right)\end{array}\right]_{j} \cdot z$.
Corresponding to Eq. (2) and Figure 4, indices 1 and 2 are now replaced by a and b. Due to the mentioned correlation between $V_{z}$ and $\mathrm{u}_{\mathrm{v}}$, the application of Eq. (19) is simplified by introducing $V_{z}=1$ and the associated unit shear deformations $\mathrm{u}_{\mathrm{V}}\left(V_{z}=1\right)$ :

$$
\begin{aligned}
& \sigma_{x}=\frac{M_{y}}{I_{y}} \cdot z-E \cdot q_{z} \cdot u_{V}\left(V_{z}=1\right)+ \\
& \frac{E}{I_{y}} \cdot q_{z} \cdot \sum_{j=1}^{n_{e}}\left[\begin{array}{l}
\frac{t \cdot \ell}{6} \cdot\left[\begin{array}{l}
2 \cdot z_{a}+z_{b} \\
z_{a}+2 \cdot z_{b}
\end{array}\right] \cdot\left[\begin{array}{l}
u_{V, a}\left(V_{z}=1\right) \\
u_{V, b}\left(V_{z}=1\right)
\end{array}\right]+ \\
\frac{t \cdot \ell^{3}}{180 \cdot G \cdot I_{y}} \cdot\left(4 \cdot z_{a}^{2}+7 \cdot z_{a} \cdot z_{b}+4 \cdot z_{b}^{2}\right)
\end{array}\right]_{j} \cdot z .
\end{aligned}
$$

Equations (19) and (20), respectively, describe the behaviour in-span of a beam appropriately (in sense of beam theory). They can also be applied to beams subjected to continuous triangular or trapezoidal loads as a very good approximation, where in the equations $\mathrm{q}_{\mathrm{z}}$ should then be regarded with the mean value of the distributed load. However, using this approach for shear force developments at points of discontinuity (point loads, supports), descriptions using beam theory are complicated not to say problematical, since shear displacements would also be approximated discontinuously and thus unrealistic. For that reason, additional investigations and analyses are necessary, not be covered in this paper though. It should only be mentioned that in regions of point loads, a distinct two-dimensional stress state occurs in the member flange, strongly influencing the deformation behaviour $\mathrm{u}$ and therefore stresses $\sigma_{\mathrm{x}}$ in longitudinal beam direction. Since these stress states do not directly apply and correspond to beam theory anymore, an instantaneous capture and approximation in the equations presented here cannot be provided.

\section{Implementation and validation}

The finite element procedure shown in section 2 and the stress distribution described in section 3 have been implemented in a corresponding software (Kraus 2016). The solution of the approach is exemplarily shown in the following and compared to finite element analysis using shell elements (RFEM 5) as well as the solution gained by the method of effective widths for elastic shear lag according to Eurocode 3 (EN 1993-15: 2006 + AC: 2009). 

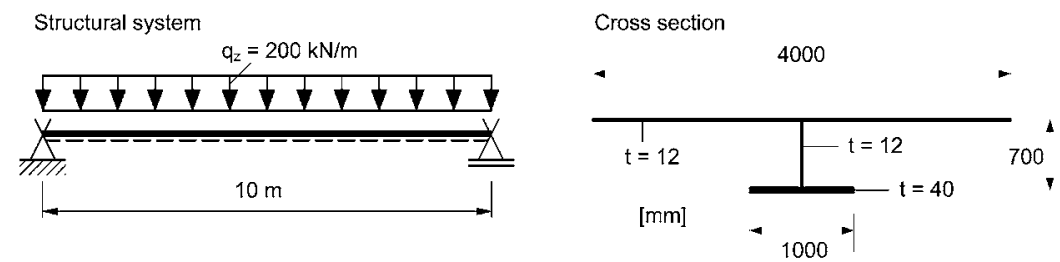

Fig. 8. Structural system and cross section of the example

Figure 8 shows the structural system and the cross section being analysed. The cross section is discretized using finite elements of Eq. (2) and the solution of the equation system (3) is shown in Figure 9(a) taking the rigid-body ratio according to Eq. (9) into account. Normal stresses are determined using Eq. (19) and the outcome calculated with (Kraus 2016) is shown in Figure $9(\mathrm{~b})$. The minimum normal stress is determined to $\sigma_{\mathrm{x}}=-9.17 \mathrm{kN} / \mathrm{cm}^{2}$ in the upper flange and $\sigma_{\mathrm{x}}=$ $-6.16 \mathrm{kN} / \mathrm{cm}^{2}$ at the flange edges.

For reasons of comparison, Figure 10 shows the calculation results using finite shell elements (RFEM 5). With $\sigma_{\mathrm{x}}=-9.25 \mathrm{kN} / \mathrm{cm}^{2}$ and $\sigma_{\mathrm{x}}=-5.99 \mathrm{kN} / \mathrm{cm}^{2}$ in the upper flange a good compliance to the proposed solution provided in Figure 9 with small deviations becomes apparent for this example.

The stresses are also compared to the solution gained by the effective width method according to Eurocode 3. Considering the beam length of $L_{e}=$ $1000 \mathrm{~cm}$, it is: upper flange:

$$
\begin{aligned}
& b_{0}=200 \mathrm{~cm}, \kappa=\alpha_{0} \cdot b_{0} / L_{e}=1.0 \cdot 200 / 1000=0.2 \Rightarrow \\
& \beta=1 /\left(1+6.4 \cdot \kappa^{2}\right)=0.796 \Rightarrow b_{e f f}=\beta \cdot b_{0}=159 \mathrm{~cm} ;
\end{aligned}
$$

lower flange:

$$
\begin{aligned}
& b_{0}=50 \mathrm{~cm}, \kappa=\alpha_{0} \cdot b_{0} / L_{e}=1.0 \cdot 50 / 1000=0.05 \Rightarrow \\
& \beta=1 /\left(1+6.4 \cdot \kappa^{2}\right)=0.984 \Rightarrow b_{\text {eff }}=\beta \cdot b_{0}=49.2 \mathrm{~cm} .
\end{aligned}
$$

Modelling the cross section and regarding the flange widths of $2 \cdot 159=318 \mathrm{~cm}$ (instead of $400 \mathrm{~cm}$ ) and $2 \cdot 49.2=98.4 \mathrm{~cm}$ (instead of $100 \mathrm{~cm}$ ) leads the following properties, where the web height is modelled to the mid-lines of the flanges for comparative reasons:

$$
\begin{aligned}
& \text { Second moment of inertia: } \quad I_{y}=984000 \mathrm{~cm}^{4} \text {; } \\
& \begin{array}{l}
\text { Position of centre of gravity: } \\
\text { (measured from the upper flange) }
\end{array} z_{S}=35.5 \mathrm{~cm} .
\end{aligned}
$$
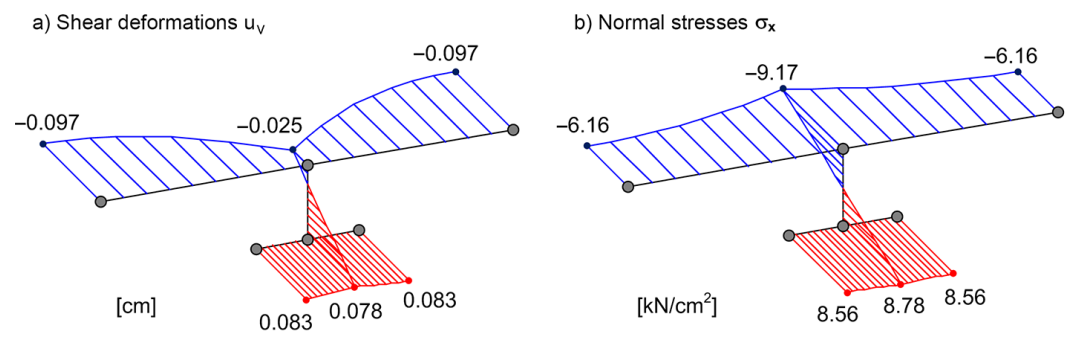

Fig. 9. Shear deformations (at supports) and stress distribution (at midspan) of the presented approach

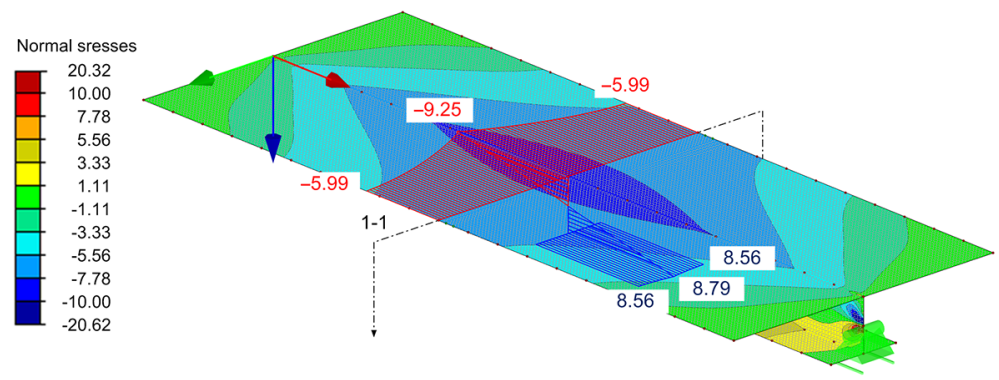

Fig. 10. Stress distributions using finite element shell calculations (RFEM 5) 
The stresses in the upper flange as defined in Figure 11 result to:

$$
\begin{aligned}
\sigma_{1}= & M_{y} / I_{y} \cdot z=250000 / 984000 \cdot 35.5=9.02 \mathrm{kN} / \mathrm{cm}^{2} ; \\
\sigma_{2}= & 1.25 \cdot(\beta-0.2) \cdot \sigma_{1}=1.25 \cdot(0.796-0.2) . \\
& 9.02=6.72 \mathrm{kN} / \mathrm{cm}^{2}
\end{aligned}
$$

The comparison with Figures 9 and 10, respectively, illustrates that the maximum stress approximation regarding the effective width method is quite similar. However, further calculations show, that differences between the three methods rise with an increase of flange width. In the performed computations, the solution of the method presented in this paper always lead to stresses, which were located between the solutions of the finite shell element analysis and the method of effective width. From the comparative investigations it can also be stated, that at least up to flange widths of $0.5 \cdot L_{e}$, calculations of the presented approach show a good agreement to the finite element shell calculations. Investigations regarding non-prismatic beams have not been performed so far, since consequential influences, which might occur, are also not covered by formulations of Eurocode 3 (EN 1993-1-5:2006 + AC: 2009). This issue is to be picked up in prospective analyses.

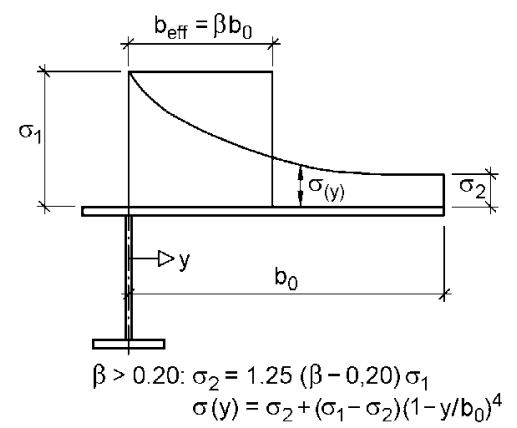

Fig. 11. Distribution of longitudinal stresses in the upper flange (EN 1993-1-5: 2006 + AC: 2009)

\section{Conclusions}

In this paper a numerical approach based on beam theory for the identification of normal stresses caused by bending moments regarding effects of elastic shear lag is presented. For that purpose, one-dimensional finite elements for the modelling of cross sections are specified, capturing their shear deformation behaviour. The influence is subsequently considered in the normal stress distribution and the proposed procedure is exemplarily put to use, showing good compliance to approximations of shell finite element formulations and the method of effective width according to Eurocode 3 . The approach can be employed to the stress calculation in-span of beams subjected to distributed loads not influenced by point loads or support reactions very well. For applications to continuous beams, additional investigations going beyond beam theory are necessary.

\section{Disclosure statement}

The author does not have any competing financial, professional, or personal interests from other parties.

\section{References}

EN 1993-1-5: $2006+$ AC: 2009. Eurocode 3: Design of steel structures - Part 1-5: Plated structural elements. European Standard.

Kindmann, R.; Kraus, M. 2011. Steel Structures - Design using FEM. Berlin: Ernst \& Sohn (Wiley) publishing.

Kindmann, R.; Krahwinkel, M. 2016. Stahl- und Verbundkonstruktionen. $3^{\text {rd }}$ ed. Wiesbaden: Springer Vieweg publishing.

Kraus, M. 2005. Computerorientierte Berechnungsverfahren für beliebige Stabquerschnitte des Stahlbaus. Aachen: Shaker publishing.

Kraus, M. 2016. QSW-FE ML. Finite Element Program for cross sections using one-dimensional elements. Edition of the Chair of Steel and Hybrid Structures, Bauhaus-University Weimar.

RFEM 5 (Release 5.03.0011). Dlubal Software GmbH.

Schmidt, H.; Peil, U. 1976. Berechnung von Balken mit breiten Gurten - Tafeln zur Ermittlung des voll mitwirkenden Gurtquerschnittes und der Gurtspannungsverteilung. Berlin, Heidelberg, New York: Springer publishing. https://doi.org/10.1007/978-3-642-51892-8

Schmidt, H.; Peil, U.; Born, W. 1979. Scheibenwirkung breiter Straßenbrückengurte - Verbesserungsvorschlag für Berechnungsvorschriften (mitwirkende Gurtbreite), Bauingenieur 54: 131-138.

Sedlacek, G.; Bild, S. 1990. Vorgehensweise bei der Ermittlung der mittragenden Breite, Bauingenieur 65: 551-562.

Matthias KRAUS. The author studied civil engineering at the University of Technology Darmstadt. In 2001 he changed to the University of Bochum as researcher, where he received his doctoral degree in 2005 and then worked as chief engineer at the Institute of Steel and Composite Structures. Between 2010 and 2015 he worked at Schürmann-Kindmann and Partners Consulting Engineers as chief engineer and he had adjunct lectureships at the University of Bochum and the Vietnamese-German University in Ho-Chi-Minh City. In 2015 he was appointed to a full professorship and the head of the Chair of Steel and Hybrid Structures at the Bauhaus-University Weimar. 